\title{
Espectroscopia de admitancias de banda ancha aplicada al control microestructural de propiedades eléctricas en varistores cerámicos
}

\author{
FERNÁNDEZ HEVIA, A. C. CABALLERO*, J. DE FRUTOS, J. F. FERNÁNDEZ*
}

Escuela Técnica Superior de Ingenieros de Telecomunicación, Universidad Politécnica de Madrid, Ciudad Universitaria s/n, 28040 Madrid, España * Departamento de Electrocerámica, Instituto de Cerámica y Vidrio, CSIC, 28049 Camino de Valdelatas s/n, Campus de Cantoblanco, Madrid, España

En el presente estudio se utiliza la teoría de transporte de carga a través de interfases eléctricamente activas para estudiar las propiedades electrónicas de varistores basados en $\mathrm{ZnO}$. Se obtiene un rango de temperaturas de sinterización en el que la respuesta eléctrica microscópica del material se mantiene esencialmente constante, de manera que su respuesta macroscópica está controlada por su microestructura. Esto permite una gran precisión en el control de las propiedades eléctricas funcionales del material.

\section{Palabras clave:}

\begin{abstract}
Application of broadband admitance spectroscopy to microstructure control of the electrical properties of ceramic varistors.
\end{abstract}
In the present work, the theory of charge transport through active interfaces is used to study the electronic properties of $\mathrm{ZnO}$-based varistors. It has been observed that for a certain range of sintering temperatures the microscopic electrical response of the material is constant, so that the macroscopic response is microstructure controlled. This allows controlling the functional response of the material with a high precision.

Keywords:

\section{INTRODUCCIÓN}

Los equipos electrónicos son muy sensibles a la estabilidad eléctrica de sus circuitos de alimentación y, por tanto, muy vulnerables ante las sobretensiones transitorias que se producen en las redes eléctricas [1]. Por ello, los dispositivos de protección frente a sobretensiones han cobrado gran importancia a la hora de garantizar la fiabilidad del complejo entramado tecnológico moderno. Los varistores son semiconductores cerámicos con interfaces eléctricamente activas, cuyas propiedades de transporte de carga extremadamente no lineales [2,3] les permiten ejercer una protección eficaz contra los fenómenos de sobretensión $[4,5]$.

La funcionalidad de los varistores depende de la no linealidad de su característica I-V, la cual depende de la interacción entre dos aspectos básicos del material: su estructura electrónica y su morfología microscópica o microestructura. En la fabricación industrial de varistores, es sabido que existen rangos de temperatura de sinterización en los que la respuesta eléctrica macroscópica parece quedar totalmente determinada por la microestructura. La existencia de estos rangos es muy útil pues otorga robustez al proceso industrial y permite obtener propiedades eléctricas predefinidas mediante la aplicación de técnicas de control microestructural, las cuales han alcanzado un elevado nivel de precisión. Sin embargo, no existen estudios sobre las razones últimas de este tipo de comportamiento: el presente trabajo pretende estudiar la estructura electrónica de un material varistor sobre dicho rango de temperaturas de sinterización, a fin de encontrar una explicación para el control puramente microestructural de la respuesta eléctrica en dichas regiones.

\section{MODELO DE DOBLE BARRERA SCHOTTKY}

La estructura electrónica de semiconductores policristalinos con bordes de grano activos $[2,3,6,7]$ se describe mediante un modelo de doble barrera Schottky (DBS) que interrelaciona tres regiones microscópicas (véase Fig. 1): (1) la interfaz o borde de grano (BG), caracterizado por una densidad de estados electrónicos superficiales de naturaleza aceptora $\mathrm{N}_{s}(E)$, con secciones eficaces de captura $\sigma_{s}(E)$; (2) la región de carga espacial (RCE), caracterizada por un número arbitrario $d$ de estados profundos, descritos por su concentración $\mathrm{N}_{\mathrm{v}^{\prime}}$ su nivel energético $\xi_{v^{\prime}}$ y su sección eficaz de captura $\sigma_{v}(v=1 \ldots d$, numerados de más someros a más profundos); y (3) el interior de grano (IG), caracterizado por un donador somero (responsable de la conductividad tipo-n del $\mathrm{ZnO}$ ), descrito mediante su concentración $\mathrm{N}_{0}$ y nivel energético $\xi_{0}$ (véase Fig. 1). La Fig. 1 muestra una típica estructura de DBS: $E_{C}(x, V)=-q_{e} \times \Phi(x, V)$ es la geometría de la banda de conducción bajo una diferencia de potencial $V=V_{0^{\prime}} \Phi(\mathrm{x}, \mathrm{V})$ es el potencial electrostático, y $\Phi_{B}(V) \equiv-\left.\Phi(x, V)\right|_{\mathrm{x}=0}$ es la altura de la barrera. La concentración de portadores en la banda de conducción, función de $N_{0}$ y $\xi_{0^{\prime}}$ define la posición del nivel de Fermi en el interior de los cristales $E_{F}$.

\section{TÉCNICAS EXPERIMENTALES}

\subsection{Fundamentos.}

En régimen estacionario (bajo una diferencia de potencial $V=V_{0}$ constante), el transporte de carga a través de bordes de grano activos se describe mediante la teoría de Bethe de emisión termoiónica, extendida para incorporar los procesos de captura/emisión de portadores en BG [6]. La densidad de corriente viene dada por

$$
J_{0} \equiv J_{0}\left(V_{0}, \Phi_{B 0}\right)=A^{*} T^{2} \exp \left[-\left(\xi+q_{e} \Phi_{B 0}\right) / k_{B} T\right]\left(1-\frac{c_{0}}{2}\right)\left(1-e^{-q_{e} V_{0} / k_{B} T}\right)
$$

donde el subíndice " 0 " indica régimen estacionario, $\mathrm{A}$ * es la constante de Richardson $\left(29 \mathrm{~A} / \mathrm{cm}^{2} \mathrm{~K}\right)$, 


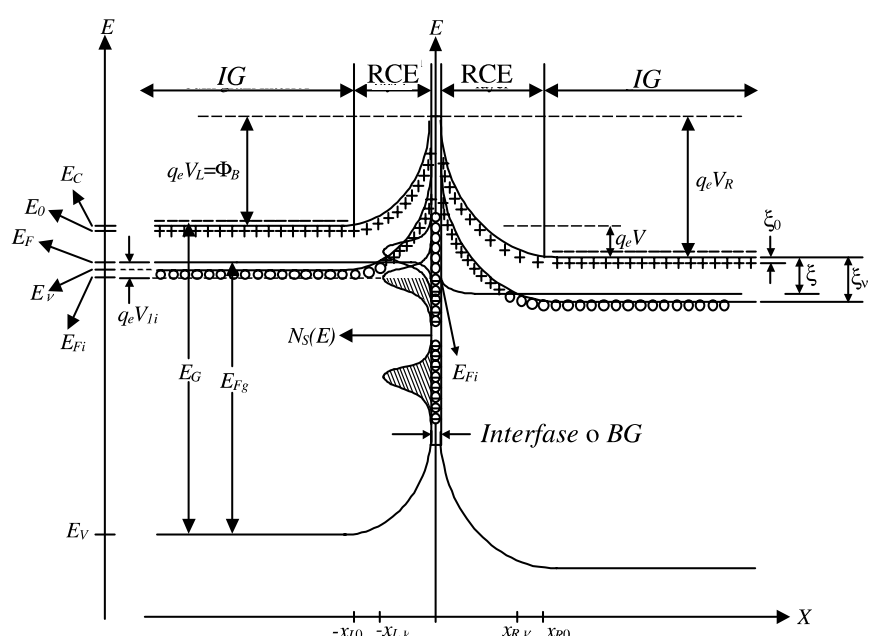

Figura 1. Esquema de bandas de la estructura electrónica de doble barrera Schottky. Los círculos vacíos significan estados neutros; los que llevan un signo "-", estados aceptores ocupados; los signos "+" representan donadores ionizados. $E_{F i}$ es la energía de Fermi en la interfaz, separada por $q_{e} V_{1 i}$ de la energía de Fermi en el interior de grano.

$$
c_{0} \equiv c_{0}(V)=\int \sigma_{S}(E) N_{S}(E) \times\left[1-f_{F}\left(E, E_{F i}\right)\right] d E
$$

es un coeficiente de captura que describe la "voracidad" de la interfaz, $f_{F}\left(E, E_{F i}\right)$ es la función de distribución de Fermi, $E_{F i}$ es el cuasi-nivel de Fermi en la interfaz [6,7], y

$$
\begin{gathered}
\Phi_{B}(V)=\frac{V_{C}}{4}\left(1-\frac{V}{V_{C}}\right)^{2}+\frac{1}{q_{e} \gamma} \sum_{v=1}^{d} \gamma_{v}\left(\xi_{v}-\xi\right) \\
V_{C}=\frac{1}{2 \gamma}\left(\frac{Q_{S}}{\varepsilon_{0} \varepsilon_{r}}\right)^{2}
\end{gathered}
$$

es la altura de la barrera, con

$$
\gamma_{\mathrm{v}}=\frac{q_{e} N_{\mathrm{v}}}{\varepsilon_{0} \varepsilon_{r}}, \quad \gamma=\sum_{\mathrm{v}=0}^{d} \gamma_{\mathrm{v}}
$$

y donde $Q_{s}$ es la carga total atrapada en la interfaz. Tanto $\mathrm{c}_{0}^{\wedge}$ como el segundo sumando de la Ec. (1) se tendrán en cuenta en el presente trabajo, a pesar de haber sido sistemáticamente despreciados en la literatura. En lo referente a $\mathrm{c}_{{ }^{\prime}}{ }^{\prime}$ los estudios originales de Seager y Pike $[8,9]$ sobre Si policristalino parecen justificar la aproximación $\hat{c}_{0} \approx 0$ : valores típicos de $\sigma_{\tilde{S}} \sim 10^{-14} \mathrm{~cm}^{2}$ y de $N_{S} \sim 10^{12} \mathrm{~cm}^{-2}[6,8,9]$, llevan a $\mathrm{c}_{0} \approx 10^{-2}$. Sin embargo, para materiales varistores, cuya propia funcionalidad requiere elevadas densidades de estados superficiales por encima del nivel de Fermi, se han medido valores típicos $N_{s} \sim 10^{13}-10^{14} \mathrm{~cm}^{-2}$ y $\sigma_{s} \sim 10^{-13}$ $10^{-14} \mathrm{~cm}^{2}[3,6,7]$ que llevan a $\hat{c}_{0} \approx 1$, en cuyo caso la aproximación es claramente injustificable.. Con respecto al segundo sumando de la Ec. (6), constituye la contribución directa de los niveles profundos a $\Phi_{B}$ y su contribución a la altura total de la barrera es cuantitativa $(\approx 0.4 \mathrm{eV})$ y cualitativamente relevante.

En régimen no estacionario [6-9], bajo una excitación $V \equiv V(t) \equiv V_{0}+\Delta V=V_{0}+\widetilde{V} e^{i \omega t}\left(\right.$ con $V_{0}$ constante y arbitrario y $\left.\tilde{\mathrm{V}}<<\mathrm{k}_{\mathrm{B}} \mathrm{T}\right)$, obtenemos el siguiente conjunto de ecuaciones para la admitancia $\mathrm{Y}\left(\mathrm{V}_{0^{\prime}} \omega\right)=\mathrm{G}\left(\mathrm{V}_{0^{\prime}} \omega\right)+\mathrm{i} \omega \mathrm{C}\left(\mathrm{V}_{0^{\prime}} \omega\right)$ de una doble barrera Schottky:

donde

$$
V \equiv V(t) \equiv V_{0}+\Delta V=V_{0}+\widetilde{V} e^{i \omega t}
$$

$$
\begin{aligned}
& G\left(V_{0}, \omega\right)=G_{0}+\omega \operatorname{Im}\left\{C_{s}\right\}-\frac{\omega C_{R}}{C_{L}+C_{R}} \operatorname{Im}\left\{C_{Q}\right\} \\
& +\left(\frac{q_{e} J_{0}}{k_{B} T\left(C_{L}+C_{R}\right)}-\frac{1}{2 \tau}\left(\sigma_{S F}+\frac{2}{1+e^{-q_{e} V_{0} / k_{B} T}}+\frac{q_{e}^{2} N_{T 1}\left(E_{F}\right)}{C_{L}+C_{R}}\right)\right)\left[C_{Q 0}-\operatorname{Re}\left\{C_{Q}\right\}\right]
\end{aligned}
$$

$$
\begin{gathered}
\omega C\left(V_{0}, \omega\right)=\omega \operatorname{Re}\left\{C_{s}\right\}-\frac{\omega C_{R}}{C_{L}+C_{R}} \operatorname{Re}\left\{C_{Q}\right\} \\
+\left(\frac{1}{2 \tau}\left(\sigma_{S F}+\frac{2}{1+e^{-q_{e} V_{0} / k_{B} T}}+\frac{q_{e}^{2} N_{T 1}\left(E_{F}\right)}{C_{L}+C_{R}}\right)-\frac{q_{e} J_{0}}{k_{B} T\left(C_{L}+C_{R}\right)}\right) \operatorname{Im}\left\{C_{Q}\right\} \\
\sigma_{S F}=\left.\sigma_{S}(E)\right|_{E=E_{F i}}
\end{gathered}
$$

es la sección eficaz de captura de los estados de interfaz localmente activos alrededor del cuasi-nivel de Fermi, $C_{0} \equiv C_{Q}\left(V_{0}, \omega\right)$ es una cantidad compleja que contiene toda la información sobre $N_{S}(E)$ y representa la respuesta eléctrica de la interfaz [6], $C_{R} \equiv C_{R}\left(V_{0^{\prime}} \omega\right)$ y $C_{L} \equiv C_{L}\left(V_{0^{\prime}} \omega\right)$ son capacidades complejas asociadas con la estructura electrónica de la región de carga espacial [7], $C_{\mathrm{s}} \equiv C_{s}\left(V_{0^{\prime}}(\omega)=C_{\mathrm{L}} C_{\mathrm{R}} /\left(C_{\mathrm{L}}+C_{\mathrm{R}}\right)\right.$, y $C_{\mathrm{Q} 0} \equiv C_{\mathrm{Q0}}\left(V_{0}\right)$, $C_{R 0} \equiv C_{R 0}\left(V_{0}\right)$ y $C_{L 0} \equiv C_{L 0}\left(V_{0}\right)$ son los límites estacionarios de $C_{Q^{\prime}} C_{R}$ y $C_{L}$ respectivamente, v.g.:

$$
C_{Q 0} \equiv C_{Q 0}\left(V_{0}\right)=\lim _{\omega \rightarrow 0} C_{Q}\left(V_{0}, \omega\right)
$$

Por último $G_{0} \equiv G_{0}\left(V_{0}\right)$ es la conductividad en régimen estacionario

$$
\begin{aligned}
G_{0}\left(V_{0}\right) & =\left\{\frac{q_{e} J_{0}}{k_{B} T\left(C_{L 0}+C_{R 0}\right)}-\frac{q_{e}^{2} N_{T 1}\left(E_{F}\right) / 2 \tau}{C_{L 0}+C_{R 0}}\right\}\left(C_{R 0}-C_{Q 0}\right) \\
& +\left(\sigma_{S F}+\frac{2}{1+e^{-q_{e} V_{0} / k_{B} T}}\right) \frac{C_{Q}(0)}{2 \tau}+\frac{e A}{k_{B} T} e^{-\left(\xi+q_{e} \Phi_{B 0}+q_{e} V_{0}\right) / k_{B} T}
\end{aligned}
$$

Las respuestas eléctricas del BG y de la RCE están fuertemente acopladas y no son representables, ni juntas ni por separado, como combinación de elementos de circuito: la obtención de propiedades físicas microscópicas requiere la cuidadosa exploración de determinados límites de las Ecs. (5)-(6). En particular, el límite de baja frecuencia $[10,11]$ proporciona información sobre $\mathrm{N}_{\mathrm{s}}(\mathrm{E})$ y $\sigma_{\mathrm{s}}(\mathrm{E})$, i.e., sobre la estructura electrónica del BG, mientras que el límite de campo nulo y alta frecuencia [12] proporciona información sobre $\mathrm{N}_{0^{\prime}} \xi, \mathrm{y} \xi_{0^{\prime}}$ i.e., sobre la estructura electrónica del IG.

\subsection{Procedimiento}

El estudio de la estructura electrónica se realizó ajustando la respuesta eléctrica experimental (i.e., la admitancia) a las expresiones analíticas presentadas en la sección anterior, en las que todas las propiedades físicas microscópicas asociados con la DBS aparecen como parámetros ajustables: para un conjunto determinado de densidades, energías y secciones eficaces de captura de los niveles profundos, densidad y energía del donador somero, y densidad y sección eficaz de captura de los estados de interfaz, la respuesta eléctrica del material puede determinarse completamente y, por tanto, ajustarse a un conjunto suficiente de curvas espectrales experimentales correspondientes a distintas temperaturas y campos aplicados. El procedimiento experimental viene dictado por el hecho de que, a campo nulo, la respuesta eléctrica de la interfaz desaparece $[2,6]$ y la respuesta de la RCE no depende de los detalles de la densidad de estados en BG, haciendo posible la exploración de la RCE sin necesidad de conocer dichos detalles. Por esta razón, la secuencia de estudio es:

1. Estudio espectroscópico del IG, en el límite de altas frecuencias y campo nulo, según la técnica desarrollada por Fernández Hevia et al. [12].

2. Estudio espectroscópico de la RCE mediante ajuste de la respuesta espectral de banda ancha, a campo nulo sobre y con temperatura variable, a los correspondientes límites $\left(V_{0}=0\right)$ de las Ecs. (5)-(6).

3. Estudio espectroscópico del BG, en el límite de baja frecuencia, según técnicas desarrolladas por Fernández Hevia et al. $[10,11]$. 
Para llevar a cabo los ajustes precedentes, es necesario escoger una forma parametrizable para las funciones $N_{s}(E)$ y $\sigma_{s}(E)$. En nuestro caso, basándonos en resultados preliminares [11], hemos asumido $N_{S}(E)=N_{0}(E)+N_{1}(E)+N_{2}(E)$, donde $N_{1}$ y $N_{2}$ son gausianas centradas sobre el nivel de Fermi en equilibrio, y parametrizadas por su área total, su anchura y la posición de su centro en el gap con respecto al fondo de la banda de conducción. $N_{0}$ es otra gausiana, pero situada bajo el nivel de Fermi en equilibrio, de manera que la única información accesible es la carga total atrapada en ese rango de energías [11]; por tal motivo $N_{0}$ aporta un único parámetro, por ejemplo su área total:

$$
N_{S}(E)=\frac{N_{S 0}}{\sqrt{2 \pi}} e^{-\frac{(E-2)^{2}}{2}}+\frac{N_{S 1}}{\sqrt{2 \pi} \Sigma_{1}} e^{-\frac{\left(E-E_{S 1}\right)^{2}}{2 \Sigma_{1}^{2}}}+\frac{N_{S 2}}{\sqrt{2 \pi} \Sigma_{2}} e^{-\frac{\left(E-E_{S 2}\right)^{2}}{2 \Sigma_{2}^{2}}}
$$

donde las energías se miden respecto al fondo de la banda de conducción. Por último:

$$
\sigma_{S}(E)=\frac{\sigma_{S 1} N_{S 1}(E)+\sigma_{S 2} N_{S 2}(E)}{N_{S 1}(E)+N_{S 2}(E)}
$$

con $\sigma_{S 1}$ y $\sigma_{S 2}$ constantes.

La Fig. 2 muestra la evolución espectral de la resistividad serie, que es el parámetro eléctrico utilizado en la región de microondas $(\sim 1 \mathrm{GHz})$ [12] para estudiar la estructura electrónica del IG. Como puede verse, las medidas obtenidas con distintos equipos en los diferentes rangos espectrales se solapan con gran precisión. Para el análisis de la estructura electrónica del BG y la RCE, se utiliza el término de pérdidas mostrado en la Fig. 3. Las verdaderas pérdidas dieléctricas permiten, por ajuste de su variación con la temperatura a campo nulo, determinar los parámetros asociados con la RCE (concentraciones $\mathrm{N}_{\mathrm{v}^{\prime}}$, niveles de energía $\xi_{v^{\prime}}$ y secciones eficaces de captura $\sigma_{v}$ de los estados profundos). Las pérdidas por conducción $\left(G_{0} / \omega\right)$ permiten, por ajuste a la Ec. (8) de su variación con el campo externo $V_{0}$ y con la temperatura [11], determinar los parámetros asociados con el BG, $\mathrm{N}_{\mathrm{s}}(\mathrm{E})$ y $\sigma_{\mathrm{s}}(\mathrm{E})$.

\subsection{Muestras}

Para las medidas de baja y media frecuencia, las muestras utilizadas fueron discos de $1.5 \mathrm{~mm}$ de altura y $15 \mathrm{~mm}$ de diámetro elec- trodazos con Ag, cortados de varistores comerciales de alta tensión sinterizados a temperaturas de $1160^{\circ} \mathrm{C}, 1180^{\circ} \mathrm{C}$, y $1200^{\circ} \mathrm{C}$. Para su caracterización eléctrica se aplicaron electrodos de plata sinterizados en aire a $160^{\circ} \mathrm{C}$ durante $2 \mathrm{~h}$. Para las medidas a alta frecuencia (por encima de $100 \mathrm{MHz}$ ), se mecanizaron cuidadosamente muestras en forma de pequeñas barras cilíndricas de $1.5 \mathrm{~mm}$ de diámetro por $4 \mathrm{~mm}$ de altura [12]. Esto es necesario para reducir los efectos de la resonancia geométrica RCL [12], minimizar el efecto pelicular en los electrodos, incrementar la penetración de campo hacia el interior del cuerpo cerámico y asegurar la transversalidad del campo electromagnético que se propaga en el material.

\section{RESULTADOS}

\subsection{Interior de grano}

Por aplicación directa de la técnica espectroscópica presentada en [12], se obtuvieron los parámetros asociados al IG: la concentración $\mathrm{N}_{0}$ y nivel de energía $\xi_{0}$ del donador somero, así como el nivel de Fermi en los granos $\xi$. Los resultados correspondientes se muestran en la Tabla 1 .

TABLA I. PROPIEDADES ELECTRÓNICAS DEL DONADOR SOMERO EN LOS TRES TIPOS DE MUESTRAS ESTUDIADAS.

\begin{tabular}{|c|c|c|c|}
\hline \multirow{2}{*}{$\begin{array}{c}\text { Temperatura de } \\
\text { sinterización }\end{array}$} & $\begin{array}{c}\text { Nivel de energía } \\
\left(\xi_{0}\right)\end{array}$ & $\begin{array}{c}\text { Concentración de } \\
\text { donadores }\left(\mathrm{N}_{0}\right)\end{array}$ & $\begin{array}{c}\text { Nivel de Fermi } \\
\text { en equilibrio }(\xi)\end{array}$ \\
\cline { 2 - 4 } & $\mathrm{eV}$ & $\mathrm{cm}^{-3}$ & $\mathrm{eV}$ \\
\hline $1160^{\circ} \mathrm{C}$ & $0.05 \pm 0.01 \mathrm{eV}$ & $2.0 \times 10^{17} \mathrm{~cm}^{-3}$ & $0.15 \pm 0.02 \mathrm{eV}$ \\
\hline $1180^{\circ} \mathrm{C}$ & $0.04 \pm 0.01 \mathrm{eV}$ & $-2.0 \times 10^{17} \mathrm{~cm}^{-3}$ & $0.14 \pm 0.02 \mathrm{eV}$ \\
\hline $1200^{\circ} \mathrm{C}$ & $0.04 \pm 0.01 \mathrm{eV}$ & $-2.0 \times 10^{17} \mathrm{~cm}^{-3}$ & $0.15 \pm 0.01 \mathrm{eV}$ \\
\hline
\end{tabular}

\subsection{Región de carga espacial}

Las curvas experimentales de pérdidas dieléctricas verdaderas (restando las pérdidas por conducción), a bias cero y varias temperaturas, se ajustaron al conjunto de ecuaciones presentadas en la sección

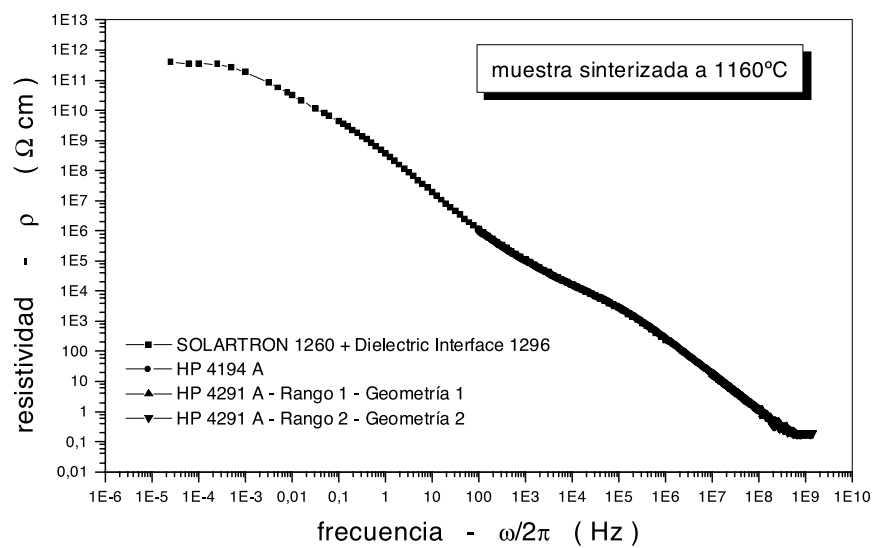

Figura 2. Resistividad serie (parte real de la impedancia) medida sobre todo el rango espectral estudiado, mostrando la convergencia a altas frecuencias y el correcto solapamiento de las medidas obtenidas con distintos equipos en diferentes intervalos de frecuencia.

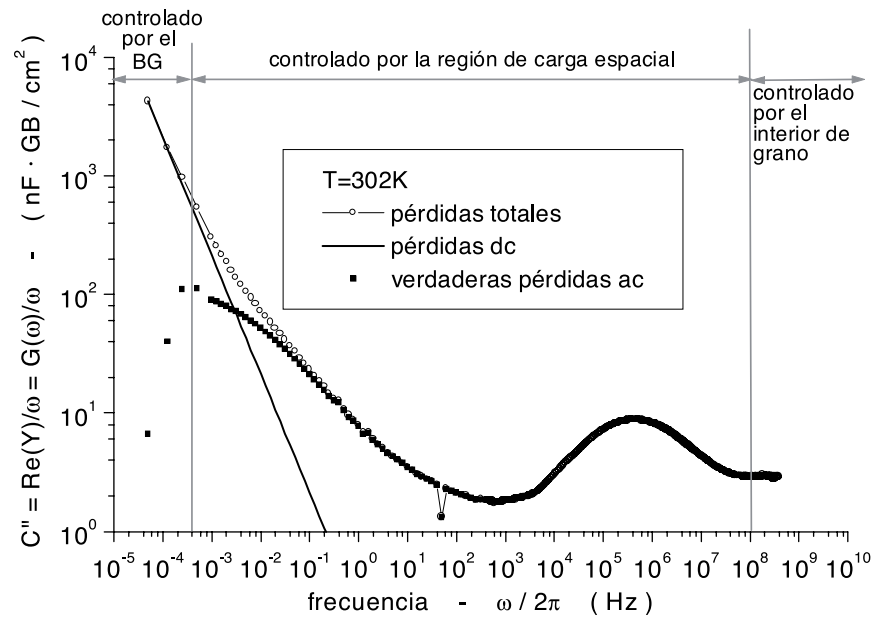

Figura 3. Término de pérdidas medido sobre un amplio rango espectral, con indicación de las regiones microscópicas que desempeñan el papel dominante en el control de la respuesta eléctrica en cada intervalos de frecuencias. Las pérdidas dc permiten el estudio del borde de grano y las verdaderas pérdidas ac (pérdidas dieléctricas) permiten estudiar la región de carga espacial. 
3.1 mediante un procedimiento iterativo que permitió obtener los valores de los parámetros físicos asociados a la RCE. Se observó una contribución dominante de las pérdidas frecuencias bajas, abordándose por tanto un ajuste sobre un rango de frecuencias restringido, acorde con la capacidad computacional disponible. La Fig. 4 muestra un ejemplo de análisis espectroscópico, correspondiente a la muestra sinterizada a $1180^{\circ} \mathrm{C}$, en el que las curvas experimentales correspondientes a varias temperaturas se han ajustado a las expresiones analíticas en el rango $\omega / 2 \pi \approx 10^{-5}-1 \mathrm{~Hz}$, en el cual se detectaron las contribuciones dominantes en todas las muestras medidas. Los resultados obtenidos fueron esencialmente idénticos en todos los casos: la Tabla 2 muestra los resultados correspondientes a la misma muestra de la Fig. 5, i.e., la que fue sinterizada a $1180^{\circ} \mathrm{C}$. Dentro de la precisión de nuestras medidas, los resultados son igualmente válidos para los otros dos tipos de muestra $\left(1160^{\circ} \mathrm{C}\right.$ y $\left.1200^{\circ} \mathrm{C}\right)$.

TABLA II. PRopiedAdeS ELECTRÓNICAS DE LA REGióN DE CARGA ESPACIAL EN LA MUESTRA SINTERIZADA A $1180^{\circ} \mathrm{C}$.

\begin{tabular}{|c|c|c|c|}
\hline \multirow{2}{*}{ Nivel No. } & Concentración $\left(\mathrm{N}_{\mathrm{v}}\right)$ & Nivel de energía $\left(\xi_{\mathrm{v}}\right)$ & Sección eficaz $\left(\sigma_{\mathrm{v}}\right)$ \\
\cline { 2 - 4 } & $\mathrm{cm}^{-3}$ & $\mathrm{eV}$ & $\mathrm{Cm}^{2}$ \\
\hline 1 & $4.8 \times 10^{16}$ & 0.41 & $1.2 \times 10^{-18}$ \\
\hline 2 & $8.5 \times 10^{16}$ & 0.47 & $2.5 \times 10^{-18}$ \\
\hline 3 & $2.0 \times 10^{17}$ & 0.51 & $2.5 \times 10^{-18}$ \\
\hline 4 & $5.0 \times 10^{17}$ & 0.56 & $1.4 \times 10^{-18}$ \\
\hline 5 & $3.6 \times 10^{18}$ & 0.60 & $1.4 \times 10^{-18}$ \\
\hline
\end{tabular}

\subsection{Borde de grano}

Por último, con toda la información recopilada en los pasos anteriores, podemos acudir al límite de baja frecuencia y, midiendo la conductividad $G_{0} \equiv G_{0}\left(V_{0} ; \mathrm{T}\right)$ a distintos campos $V_{0}$ y temperaturas $\mathrm{T}$ y ajustando los resultados a la ecuación (8) mediante una técnica iterativa [11], podemos obtener el conjunto de parámetros $N_{S 0^{\prime}} N_{S 1^{\prime}} N_{S 2^{\prime}}$ $\Sigma_{1}, \Sigma_{2^{\prime}}, \mathrm{E}_{\mathrm{S}^{\prime}}, \mathrm{E}_{\mathrm{S}^{\prime}}, \sigma_{\mathrm{S}^{\prime}}, \mathrm{y} \sigma_{\mathrm{s} 2}$ los cuales, según se explicó en la sección 3.2, determinan la densidad de estados $\mathrm{N}_{\mathrm{s}}(\mathrm{E})$ y la sección eficaz de captura $\sigma_{\mathrm{s}}(\mathrm{E})$, Ecs. (9) y (10)

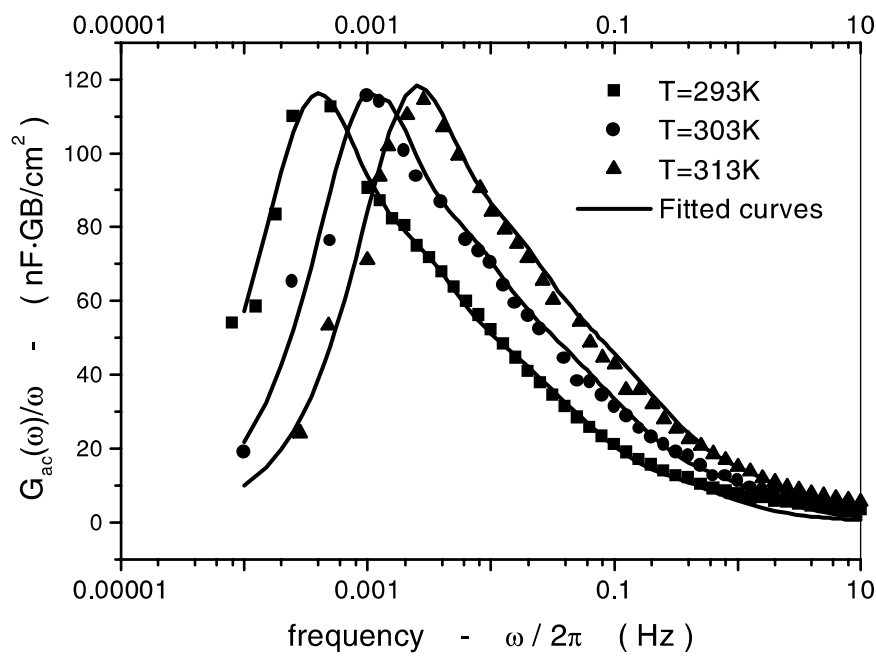

Figura 4. Puntos experimentales y curvas teóricas calculadas con los parámetros de la Tabla II, correspondientes a la muestra sinterizada a $1180^{\circ} \mathrm{C}$.
La Fig. 5 muestra el resultado de uno de estos ajustes, correspondiente a la muestra sinterizada a $1160^{\circ} \mathrm{C}$ y realizado con datos obtenidos a temperatura ambiente. La Tabla 3 resume los correspondientes parámetros obtenidos para los tres tipos de muestras bajo estudio. Vemos en dicha Tabla 3 que la tercera gaussiana no aparece en absoluto: esto se debe a que, en el rango de bias aplicados (hasta $V_{0} \approx 1.2 \mathrm{~V} / \mathrm{BG}$ ), los datos experimentales se ajustaron suficientemente bien con sólo dos gaussianas, la situada en todo momento bajo el nivel de Fermi, y otra cuya posición energética es tal que se encontraba "a medio llenar" en el estado de equilibrio termodinámico siendo, por tanto, responsable del fuerte anclaje de la barrera que presentaban las muestras [10]. La sección eficaz de captura correspondiente a la distribución observada resulto ser $\sigma_{\mathrm{S} 1}=7.2 \times 10^{-14} \pm 15 \% \mathrm{~cm}^{-2}$.

TABLA III. PROPIEDADES ELECTRÓNICAS DEL BORDE DE GRANO EN LOS TRES TIPOS DE MUESTRAS ESTUDIADAS.

\begin{tabular}{|c|c|c|c|c|}
\hline \multirow{2}{*}{$\begin{array}{c}\text { Temperatura de } \\
\text { sinterización }\end{array}$} & \multirow{2}{*}{$\mathrm{N}_{\mathrm{s} 0}$} & \multicolumn{3}{|c|}{$\mathrm{N}_{\mathrm{S1}}(\mathrm{E})$} \\
\cline { 3 - 5 } & $\left(\mathrm{cm}^{-2}\right)$ & $\mathrm{N}_{\mathrm{S} 1}$ & $\mathrm{E}_{\mathrm{S}}$ & $\Sigma_{1}$ \\
\hline$\left({ }^{\circ} \mathrm{C}\right)$ & $5.8 \times 10^{12} \pm 15 \%$ & $9.6 \times 10^{12} \pm 5 \%$ & $1.0 \pm 0.05$ & $(\mathrm{eV})$ \\
\hline 1160 & $6.2 \times 10^{12} \pm 15 \%$ & $9.2 \times 10^{12} \pm 5 \%$ & $1.0 \pm 0.05$ & $\mathrm{k}_{\mathrm{B}} \mathrm{T}$ \\
\hline 1180 & $5.6 \times 10^{12} \pm 15 \%$ & $9.3 \times 10^{12} \pm 5 \%$ & $1.0 \pm 0.05$ & $\mathrm{k}_{\mathrm{B}} \mathrm{T}$ \\
\hline 1200 &
\end{tabular}

\section{CONCLUSIÓNES}

Los resultados obtenidos demuestran que, en el rango de temperaturas de sinterización $1160^{\circ} \mathrm{C}-1200^{\circ} \mathrm{C}$, la estructura electrónica completa de los materiales varistores estudiados prácticamente no cambia. Esto explica el control puramente microestructural de la respuesta eléctrica macroscópica: en este rango de temperaturas, sólo las distintas distribuciones de tamaños de grano marcan diferencias entre las muestras. Este resultado es útil, pues permite definir este tipo de rangos a priori, durante la etapa de diseño en laboratorio de una formulación determinada, lo cual, a su vez, permite evaluar la futura robustez y flexibilidad de dicha formulación en el escalado industrial.

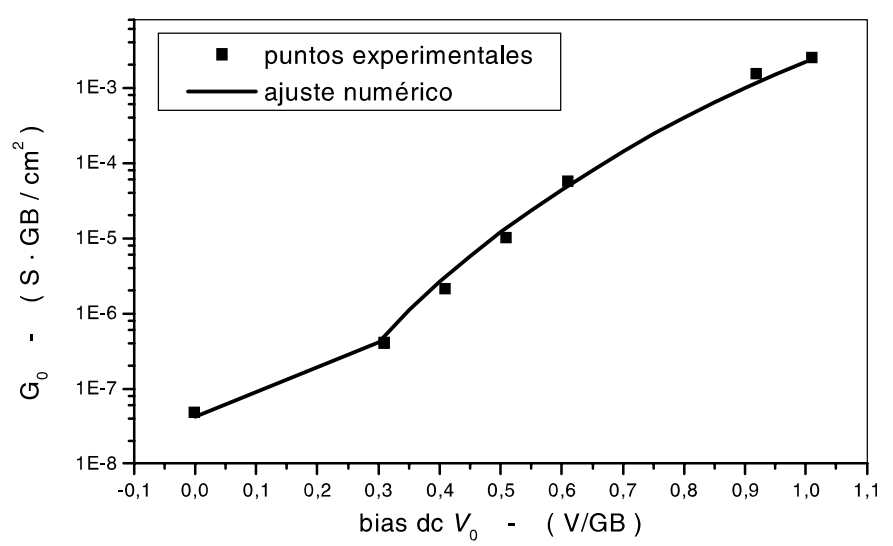

Figura 5. Puntos experimentales y curva teórica calculada con los parámetros de la Tabla III, correspondientes a la muestra sinterizada a $1160^{\circ} \mathrm{C}$ 


\section{AGRADECIMIENTOS}

Agradecemos el soporte recibido desde el CICYT, mediante el proyecto MAT2001-1682-C02-01/02.

\section{REFERENCIAS}

1. A. Greenwood, Electrical Transients in Power Systems, 2nd Edition. John Wiley \& Sons, New York 1991.

2. G. E. Pike, "Semiconducting Polycrystalline Ceramics"; pp. 731-754 in Materials Science and Technology, Vol. 11. Edited by M. V. Swain. VCH Weinheim, Germany, 1994.

3. F. Greuter, "Electrically active interfaces in $\mathrm{ZnO}$ varistors," Solid State Ionics, $\quad$ 75, 67-78 (1995).

4. T. K. Gupta, “Application of Zinc Oxide Varistors,” J. Am. Ceram. Soc., 73 [7] 1817-1840 (1990).
5. D. R. Clarke, "Varistor Ceramics," J. Am. Ceram. Soc., 82 [3] 485-502 (1999).

6. G. E. Pike, "Semiconductor grain-boundary admittance: Theory," Phys. Rev. B, 30 (2) 795-802 (1984).

7. G. Blatter and F. Greuter, "Electrical breakdown at semiconductor grain boundaries," Phys. Rev. B, 34 (12) 8555-8572 (1986).

8. C. H. Seager, G. E. Pike, and D. S. Ginley "Direct Measurement of Electron Emission from Defect States at Silicon Grain Boundaries," Phys. Rev. Lett., 43 (7), 532-535 (1979).

9. C. H. Seager and G. E. Pike, "Anomalous low-frequency grain-boundary capacitance in silicon," Appl. Phys. Lett., 37 (6), 747-749 (1980).

10. D. Fernández Hevia, J. de Frutos, A. C. Caballero, and J. F. Fernández, “MottSchottky behavior of strongly pinned double Schottky barriers and characterization of ceramic varistors," J. Appl. Phys., 92 (5) 2890-2898 (2002).

11. D. Fernández-Hevia, M. Peiteado, J. de Frutos, A. C. Caballero, and J. F. Fernández, "Wide range dielectric spectroscopy of $\mathrm{ZnO}$-based varistors as a function of sintering time," J. Europ. Ceram. Soc. (aceptado para su publicación).

12. D. Fernández Hevia, J. de Frutos, A. C. Caballero, and J. F. Fernández, “Bulkgrain resistivity and positive temperature coefficient of $\mathrm{ZnO}$-based varistors," Appl. Phys. Lett., 82 (2) 212-214 (2003). 